Nouvelles perspectives en sciences sociales

Revue internationale de systémique complexe et d'études relationnelles

\title{
Est-il bon, est-il méchant? Le rôle du nombre dans le gouvernement de la cité néolibérale
}

\section{Alain Desrosières}

Volume 7, numéro 2, mai 2012

Sur le thème de la modélisation

URI : https://id.erudit.org/iderudit/1013061ar

DOI : https://doi.org/10.7202/1013061ar

Aller au sommaire du numéro

\section{Éditeur(s)}

Prise de parole

ISSN

1712-8307 (imprimé)

1918-7475 (numérique)

Découvrir la revue

Citer cet article

Desrosières, A. (2012). Est-il bon, est-il méchant? Le rôle du nombre dans le gouvernement de la cité néolibérale. Nouvelles perspectives en sciences sociales, 7(2), 261-295. https://doi.org/10.7202/1013061ar

\section{Résumé de l'article}

Comment résoudre la contradiction entre l'ethos du statisticien et la prise en compte des rétroactions, même quand celles-ci lui apparaissent seulement comme de fâcheux obstacles à sa mission, qu'il pense être de « fournir des reflets non biaisés de la réalité » ? Il n'est pas possible d'isoler un moment de la mesure, qui serait indépendant de ses usages, et notamment des conventions qui sont la première étape de la quantification. Il faudrait désenclaver la formation des statisticiens, en la complétant par des éléments d'histoire, de sciences politiques, et de sociologie de la statistique, de l'économétrie, des probabilités, de la comptabilité et de la gestion. Ce programme, inspiré des acquis des sciences studies (Pestre, 2006), pourrait faciliter la prise en compte des outils quantitatifs dans les débats sociaux, sans verser ni dans le rejet a priori, ni dans le respect inconditionnel et naïf devant des « faits incontestables parce que quantifiés ". 


\title{
Est-il bon, est-il méchant? Le rôle du nombre dans le gouvernement de la cité néolibérale ${ }^{1}$
}

\author{
Alain Desrosières \\ Institut nationale de la statistique \\ et des études économiques (INSEE), \\ Centre Alexandre Koyré
}

Un conseiller [de Pôle Emploi] avait fini par leur expliquer les "consignes" qui leur étaient données, ici comme ailleurs, et depuis longtemps : les chiffres du chômage doivent saméliorer, quoi qu'il arrive. Cette réunion en était un des moyens. On convoque une catégorie de chômeurs, cadres, RMistes, peu importe. Une partie ne viendra pas, et sans justificatif, c'est statistique. Ils seront radiés. "Ce n'est pas grave ", avait tempéré le conseiller. Ils peuvent se réinscrire après, sills veulent, mais cela permet de faire chuter les chiffres, même pour quelques jours. Le conseiller, qui sétait mis à parler à regret, avait tout déballé, les petites combines pour masquer les chiffres, les contrats pour les collectivités avec des abattements de charges, les formules bidons pour les jeunes, ou les aides à temps partiel qui poussent l'employeur à embaucher deux mi-temps plutôt qu'un plein temps. Il disait qu'il regrettait, que ce n'était pas leur faute. Ce n'était pas lui qui truandait, c'était tout le système qui voulait ça [souligné par nous] ${ }^{2}$.

1 Ce texte est tiré d'une communication au séminaire L'informazione prima dell'informazione. Conoscenza e scelte pubbliche, Université de Milan Bicocca, 27 mai 2010. Il a été publié en Italien sous le titre «Buono o cattivo? Il ruolo del numero nel governo della città neoliberale», dans la Rassegna Italiana di sociologia, $\mathrm{n}^{\circ}$ 3, juillet-septembre 2011, p. 373-398, http://www.mulino.it/ edizioni/riviste/scheda_fascicolo.php?isbn=14761.

2 Florence Aubenas, Le Quai de Ouistreham, Paris, L'Olivier, 2010, p. 251-252. 
et extrait du beau livre de Florence Aubenas dit bien com-
ment la politique des indicateurs statistiques s'empare de la société. Elle rétroagit sur les comportements, indépendamment des souhaits des personnes concernées. Le texte qui suit cherche à mettre en perspective l'évolution historique qui a conduit à faire jouer à la statistique, naguère un outil d'émancipation, un rôle aussi funeste.

Printemps 2009 : les statisticiens français sont inquiets pour l'avenir de leur activité. Deux ans auparavant, une grave crise a éclaté entre le gouvernement et les responsables du suivi statistique du chômage. Le Directeur de l'INSEE l'(Office français de statistique) a été démis pour avoir " mal géré » cette crise. Puis, en automne 2008, a été rendue publique la décision de transférer à Metz, dans l'Est de la France, une partie de ces services. Enfin, des coupes budgétaires de grande ampleur sont annoncées. Pourtant cette statistique publique jouit encore d'une bonne réputation auprès de ses utilisateurs, acteurs économiques, journalistes, syndicalistes, enseignants, chercheurs. De nombreuses prises de position sont publiées dans la presse pour regretter ce qui est perçu comme une menace de démantèlement. La métaphore usuelle est celle de "la tentation de casser le thermomètre pour ne pas voir la hausse de la température ».

Pourtant un incident révélateur frappe une jeune statisticienne militante du "Comité de défense de la statistique publique » tout juste constitué. Participant à une manifestation des syndicats contre la politique gouvernementale, elle sollicite, par une pétition, le soutien des manifestants. Elle a la surprise de s'entendre dire : "Vos statistiques ne servent qu'à nous contrôler, à nous fliquer, à aggraver nos conditions de travail ». Par ailleurs, toujours en 2009, des universitaires, des chercheurs, des personnels de santé, s'élèvent avec force contre les " réformes " qui frappent leurs activités, et qui impliquent des évaluations quantifiées de leurs "performances ", préludes, selon eux, à une dépossession de leurs compétences spécifiques au profit de méthodes issues du "New Public Management ", reposant sur un large usage d'indicateurs quantitatifs. Un vent de révolte souffle contre cette 
quantification généralisée, chez des universitaires et des médecins. Un mouvement, l'Appel des appels, fédère ces protestations. La résistance aux évaluations quantitatives est un de ses principaux mots d'ordre.

Printemps 2009 encore : d'autres revendications, d'un tout autre style. Le gouvernement français a demandé à d'éminents économistes, Amartya Sen, Joseph Stiglitz et Jean-Paul Fitoussi de proposer des réformes des modes de calcul du Produit intérieur brut (PIB), jugé insuffisant pour quantifier la " richesse " nette créée par une nation au cours d'une année. D’autres chercheurs militants avaient de longue date anticipé cette demande très médiatisée. Ils se sont associés pour revendiquer, sous le nom de " nouveaux indicateurs de richesse ", une autre statistique, évaluant par exemple les dégâts environnementaux, le travail non payé des femmes ou les effets sociaux des inégalités ${ }^{3}$. Ce mouvement réclame d'autres quantifications, en arguant que : "Ce qui n'est pas compté ne compte pas ".

Comment penser en même temps ces deux revendications, qui portent sur des usages très différents de la quantification? Comment prendre du recul par rapport à ce qui apparaît pourtant, dans les deux cas, comme une crise de confiance contre un outil, la statistique, auparavant plutôt perçu comme une arme au service de la démocratie, à la disposition des dominés, leur permettant de dénoncer privilèges et inégalités, de critiquer les politiques injustes, de se battre pour le maintien du pouvoir d'achat? Ce côté « outil à la disposition des faibles pour combattre les puissants " a été analysé par l'historien Theodore Porter, dans son ouvrage "Trust in Numbers" ${ }^{4}$. Cette idée d'une statistique " progressiste » est largement partagée, notamment par les statisticiens publics français, qui tiennent à cet adjectif "public ", synonyme de "service public ", dépositaire de l'intérêt général, bien distinct du terme "official statistics", utilisé par les anglophones.

Jean Gadrey et Florence Jany-Catrice, Les Nouveaux indicateurs de richesse, Paris, La Découverte / Repères, 2005.

4 Theodore Porter, Trust in Numbers. The Pursuit of Objectivity in Science and Public Life, Princeton, Princeton University Press, 1995. 


\section{Les indicateurs quantitatifs rétroagissent sur les acteurs quantifiés}

Cette crise de confiance est le symptôme d'une évolution historique de long terme des relations entre des types de gouvernement de la cité, et des manières d'utiliser la quantification. Les outils quantitatifs ne sont pas seulement des outils de preuve, utilisés par les scientifiques pour étayer leurs arguments, mais aussi des outils de coordination, ou de gouvernement. Cela avait déjà été suggéré, il y plus de trente ans, par Michel Foucault ${ }^{5}$, avec son idée de gouvernementalité, puis par Porter, et aussi par Pierre Lascoumes et Patrick Le Galès, sous le titre Gouverner par les instruments ${ }^{6}$. De ce point de vue, le thème de la quantification englobe non seulement la statistique proprement dite, mais aussi la comptabilité, les indicateurs de performance, les palmarès (ou "ranking "), et tous les outils quantitatifs du New Public Management (ou NPM), maintenant bien connus des chercheurs en sciences politiques. L'analyse de Porter sur les causes et les effets de la confiance dans les nombres est convaincante, mais la récente extension de l'usage des indicateurs quantitatifs par le NPM soulève de nouvelles questions. Cela introduit une sorte de discontinuité dans l'ancien et traditionnel usage des statistiques par les gouvernements. Cet usage remonte au XVIII siècle, et a été largement développé aux XIX ${ }^{e}$ et $\mathrm{XX}^{\mathrm{e}}$ siècles.

En bref, cette discontinuité viendrait du fait que, alors que les statisticiens publics revendiquent l'objectivité et l'indépendance de leur activité (même si l'effectivité de ces principes peut être sociologiquement discutée), en revanche, les indicateurs du NPM, qui induisent des effets de rétroaction ${ }^{7}$ sur les situations

5 Michel Foucault, Sécurité, territoire, population, Cours du Collège de France (1977-1978), Paris, Gallimard/Seuil, 2004; Michel Foucault, Naissance de la biopolitique, Cours du Collège de France (1978-1979), Paris, Gallimard/Seuil, 2004.

6 Pierre Lascoumes et Patrick Le Galès (dir.), Gouverner par les instruments, Paris, Presses de Sciences Po, 2004.

7 Ce terme "rétroaction " ne fait pas référence aux théories de l'auto-organisation, ni aux "boucles de rétraction » de la cybernétique, comme l'ont suggéré deux referees. Il s'agit plutôt de prendre en compte le fait que les 
et les comportements des acteurs, relèvent de logiques cognitives, politiques et sociologiques très différentes. Ceci avait déjà été analysé de longue date, à propos de la comptabilité, par des chercheurs anglais ${ }^{8}$. Ceci ne signifie pas que les statistiques publiques n'ont pas d'effets sur les acteurs, mais : 1) l'ethos des statisticiens professionnels exclut, en principe, qu'ils les prennent en compte, et 2) s'ils existent, ces effets sont plus macrosociaux que directement individuels. Nous ne suggérons pas que les statisticiens publics sont plus " neutres » et " objectifs » que les comptables, mais qu'il est sociologiquement pertinent de distinguer les usages des diverses notions d' " objectivité " et de " réalité " impliquées dans ces différents cas, comme l'a déjà fait l'historienne des sciences Lorraine Daston' ${ }^{9}$ dans son analyse de l'histoire de la notion d' " objectivité scientifique ".

Cette évolution du rôle des indicateurs quantitatifs dans les manières de gouverner peut être inscrite dans une périodisation des formes de l'État depuis le XVIII e siècle, selon une typologie suggérant comment sont co-construits : des conceptualisations de la société et de l'économie, des modes d'action publique, et des formes de quantification et de modélisation. Après avoir suggéré une distinction de méthode entre les deux verbes quantifier et mesurer, nous rappellerons les logiques de ces cinq formes d'État $^{10}$, puis nous montrerons comment les indicateurs du NPM

indicateurs d'évaluation d'actions ou de politiques " rétroagissent " sur les acteurs quantifiés, de manière incitative, ce qui peut par exemple les conduire à en tenir compte au moment où ils remplissent des questionnaires ou des dossiers de gestion. (Ces phénomènes de " gaming and cheating " sont analysés en détail dans le livre de Michael Power: The Audit Society. Rituals of Verification, Oxford University Press, Oxford 1999, traduction française : La Société de l'audit. L'obsession du contrôle, Paris, La Découverte, 2005.)

8 Anthony Hopwood et Peter Miller (dir.), Acounting as Social and Institutional Practice, Cambridge (UK), Cambridge University Press, 1994; Christopher Hood, "Contemporary Public Management: A New Paradigm? ", Public Policy and Administration, vol. 10, $\mathrm{n}^{\circ} 2$, 1995, p. 104-117.

9 Lorraine Daston, "Objectivity and the Escape from Perspective ", Social Studies of Science, vol. 22, no 4, 1992, p. 597-618.

10 Cette typologie de cinq formes de gouvernementalité et des statistiques qui leur correspondent, est présentée plus en détail dans Alain Desrosières, "Historiciser l'action publique : l'État, le marché et les statistiques », dans Pascale Laborier et Dany Trom (dir.), Historicités de l'action publique, Paris, 
apparaissent dans la cinquième, celle de l'État néolibéral (dans les années 1980 en Grande-Bretagne, et les années 2000 en France). Ceci introduit un trouble dans le statut des statistiques publiques, et notamment sur leur "statut de réalité ». En faisant apparaître une rétroaction de la quantification sur les comportements des acteurs, on s'éloigne de l'épistémologie réaliste qui sous-tend la métrologie de la statistique publique. Ce faisant, nous ne visons pas à dénoncer quelque forme de tromperie, de manipulation, de mésusage sinon de fraude, même si cela existe bien sûr ${ }^{11}$, mais plutôt à clarifier le rôle de cette quantification selon les contextes argumentatifs et politiques.

Nous décrirons les nouveaux indicateurs utilisés dans le suivi des politiques publiques et dans le pilotage de certaines politiques de l'Union Européenne, et aussi les débats sur la flexibilité et les changements des normes de la comptabilité d'entreprise. Il se trouve en effet que, si cette idée de rétroaction est absente de la culture et de l'ethos des statisticiens, elle est en revanche omniprésente dans les pratiques des gestionnaires et des comptables et $\mathrm{a}$, de ce fait, déjà fait l'objet de nombreuses recherches théoriques et appliquées. Enfin, nous interrogerons la pertinence même de cette idée de rétroaction. Ne résulte-t-elle pas de la division du travail historiquement contingente entre professions et disciplines?

\section{Convenir, mesurer, quantifier}

Notre démarche est sociologique et non pas épistémologique. Elle implique de donner une définition non normative du verbe quantifier. Ainsi les indicateurs quantitatifs utilisés par le New Public Management sont souvent critiqués. Que signifie « mesurer

Presses universitaires de France, 2003, p. 207-221.

Les économistes utilisateurs de statistiques ont, de longue date, décrit (pour les déplorer) les «biais " résultant, selon eux, de ces effets de rétroaction. Voir Oskar Morgenstern, On the Accuracy of Economic Observations, Princeton, Princeton University Press, 1944, traduction française, L'Illusion statistique : précision et incertitude des données économiques, Paris, Dunod, 1950. Mais cette notion de biais, issue d'une métrologie réaliste, fait seulement de celuici un obstacle gênant aux "vraies mesures ". Elle interdit d'étudier sociologiquement le jeu sur la quantification, pour lui-même. 
une performance »? La rétroaction des indicateurs induit des effets pervers : les acteurs se focalisent sur l'indicateur, et non sur l'action elle-même. Or le simple fait d'utiliser le verbe mesurer renvoie implicitement à la métrologie réaliste des sciences de la nature. Aussi, il est utile de distinguer deux idées, souvent confondues, celle de quantification et celle de mesure. Le verbe quantifier est employé ici de façon neutre et dans un sens large : exprimer et faire exister sous une forme numérique ce qui, auparavant, était exprimé par des mots et non par des nombres. (Ceci est un énoncé descriptif et non pas normatif.) En revanche, l'idée de mesure, inspirée des sciences de la nature, implique que quelque chose existe déjà sous une forme mesurable selon une métrologie réaliste, comme une grandeur physique. Dans le cas des sciences sociales ou de l'évaluation des actions publiques, l'emploi immodéré du mot mesurer induit en erreur, en laissant dans l'ombre les conventions de la quantification. Le verbe quantifier, dans sa forme active (faire du nombre), suppose que soit élaborée et explicitée une série de conventions d'équivalences préalables $^{12}$, impliquant des comparaisons, des négociations, des compromis, des traductions, des inscriptions, des codages, des procédures codifiées et réplicables, et des calculs conduisant à la mise en nombre. La mesure proprement dite vient ensuite, comme mise en œuvre réglée de ces conventions. De ce point de vue, la quantification se décompose en deux moments : convenir et mesurer. Le premier, souvent méconnu des utilisateurs (notamment des économistes), est au moins aussi important que le second.

L'usage du verbe quantifier attire l'attention sur la dimension, socialement et cognitivement créatrice, de cette activité. Celle-ci ne fournit pas seulement un reflet du monde (point de vue usuel),

$\overline{12}$ Cette notion, sociale et logique, de convention d'équivalence, doit notamment à Bruno Latour, Les Microbes. Guerre et paix, suivi de Irréductions, Paris, Métailié, 1984, dans le supplément Irréductions au livre sur Louis Pasteur, et à Laurent Thévenot, "Rules and Implements : Investments in Forms ", Social Science Information, vol. 23, $\mathrm{n}^{\circ} 1,1984$, p. 1-45, version française, "Les Investissements de formes ", Cahiers du Centre d'études de l'emploi, $\mathrm{n}^{\circ} 29$, Paris, Presses universitaires de France, 1986, p. 21-71. 
mais elle le transforme, en le reconfigurant autrement. Cette distinction entre quantifier et mesurer n'est pas " relativiste " au sens péjoratif parfois attribué à ce mot. Elle vise à séparer analytiquement deux moments historiquement et socialement distincts, comme ce fut par exemple le cas pour l' "intelligence ", quand fut imaginé le "quotient intellectuel », pour "l'opinion », quand apparurent les enquêtes par sondage de type "Gallup ", ou encore les débats plus récents sur la quantification des effets de l'action publique. L'invention, au XVII siècle, de la notion de probabilité pour quantifier l'incertain au moyen d'un nombre compris entre 0 et 1 , en a été un illustre précédent.

Le soupçon de relativisme provient de ce que l'existence réelle de l'objet, antérieurement à sa mesure, est mise en doute par certains, pour qui ce serait la mesure elle-même qui créerait l'objet. L'intelligence serait « ce qui est mesuré par les tests du QI ». L'opinion serait "ce qui est mesuré par les sondages d'opinion ». La quantification, vue comme l'ensemble des conventions socialement admises et des opérations de mesure, crée une nouvelle façon de penser, de représenter, d'exprimer le monde et d'agir sur lui. La question récurrente de savoir si une statistique " reflète plus ou moins bien la réalité » est un raccourci trompeur, contaminé par le réalisme métrologique des sciences de la nature. La statistique, et plus généralement toutes les formes de quantification (par exemple probabiliste, ou comptable), transforme le monde, par son existence même, par sa diffusion et ses usages argumentatifs, scientifiques, politiques ou journalistiques. Une fois les procédures de quantification codifiées et routinisées, leurs produits sont réifiés. Ils tendent à devenir « la réalité ", de façon apparemment irréversible. Les conventions initiales sont oubliées, l'objet quantifié est naturalisé et l'emploi du verbe « mesurer » vient machinalement à l'esprit et sous la plume.

Cela reste vrai jusqu'à ce que ces "boîtes noires " soient réouvertes, lors de controverses, comme par exemple celles sur la " mesure " du chômage, ou encore du " partage volume-prix " des taux de croissance de l'économie, quand la "mesure » de la 
hausse des prix a été critiquée en raison de l'accroissement de la " qualité " des produits (cas des ordinateurs). Dans ce cas, la mesure du taux de croissance en volume (en euros constants) du PIB, rapport entre la croissance en valeur (euros courants) et le taux d'inflation, est directement affectée par ces critiques. En 1996, un rapport au Sénat américain de Michael Boskin développa cette critique de l'indice des prix à la consommation et du taux de croissance de l'économie. Il fit scandale car, bel exemple de rétroaction, ces deux taux indexent automatiquement nombre de décisions économiques : pensions de retraite, salaires, prévisions budgétaires ${ }^{13}$.

La quantification offre un langage spécifique, permettant des transferts, des comparaisons, des agrégations, des manipulations standardisées par le calcul, et des interprétations routinisées ${ }^{14}$. Elle met à la disposition des acteurs sociaux ou des chercheurs "des objets qui tiennent ", au triple sens de leur robustesse propre (résistance à la critique), de leur capacité à se combiner entre eux, et enfin de ce qu'ils " tiennent les hommes entre eux " en les incitant, ou en les contraignant, à user de ce langage à visée universaliste, plutôt que d'un autre. Cette façon de voir est différente de celle qui est revendiquée d'habitude par les sciences sociales quantitatives et, plus généralement, par les usages des outils statistiques et comptables. Les conventions de quantification sont elles-mêmes le produit de l'histoire de l'État et des modes de gouvernement. Cette histoire peut être stylisée, d'une façon certes simplificatrice, mais qui permet de contraster la gouvernementalité néolibérale par rapport aux précédentes, en ce qu'elle implique notamment un recours massif aux indicateurs de performance et au benchmarking.

13 Michael Boskin et al., Toward a More Accurate Measure of the Cost of Living: Final Report to the Senate Finance Committee, U. S. Government Printing Office, Washington (DC), 1996.

14 Alain Desrosières, Pour une sociologie historique de la quantification, Paris, Presses de l'École des mines, 2008; Alain Desrosières, Gouverner par les nombres, Paris, Presses de l'École des mines, 2008. 


\section{La rétroaction des indicateurs diffère selon les formes d'État}

Les cinq formes de gouvernementalité ne correspondent pas à une succession dans le temps, mais plutôt à une stratification successive, chaque strate conservant, englobant mais aussi transformant, les strates précédentes. Les phénomènes de rétroaction n'en sont pas absents, mais ils ne jouent pas le même rôle que dans le cas du néolibéralisme.

L'intervention directe de l'"État ingénieur " englobe des perspectives variées, allant du mercantilisme et du colbertisme (XVII ${ }^{\mathrm{e}}$ siècle) jusqu'aux " grands projets » de la France gaulliste et aux économies planifiées socialistes. Ses statistiques y sont comparables à celles d'une grande entreprise planifiant ses ateliers, ou à celles d'une armée gérant sa logistique. Les recensements démographiques, les flux de produits en quantité physique, les tableaux input-output (ou matrices de Leontief) et la comptabilité y sont essentiels ${ }^{15}$. L'État ingénieur se déploie notamment pendant les périodes de guerre, qui impliquent une centralisation organisée des forces productives ${ }^{16}$. Les ingénieurs français issus de l'École Polytechnique (une école militaire) ont été longtemps de bons représentants de cette culture technique et politique, distincte de la culture marchande du monde anglo-américain ${ }^{17}$.

Dans le cas de la planification de l'Union soviétique, deux formes de quantification très différentes se sont succédées ${ }^{18}$. Dans les années 1920, des enquêtes par sondage aléatoire sur les modes de vie des ménages ont poursuivi une tradition de statistique

15 Le débat des années 1930 sur le «calcul socialiste » et sur la possibilité d'une planification sans prix issus du marché, peut être lu comme participant du rêve d'un tel État ingénieur. Voir Bruce Caldwell, " Hayek and Socialism ", Journal of Economic Literature, $\mathrm{n}^{\circ} \mathrm{XXXV}, 1997$, p. 1856-1890.

16 Amy Dahan et Dominique Pestre (dir.), Les Sciences pour la guerre, 19401960, Paris, Éditions de l'EHESS, 2004.

17 Une réflexion de Jacques Lesourne, ancien élève de cette école, ingénieur des mines et fondateur de la Société d'Étude et de Mathématiques Appliquée (SEMA) travaillant pour l'État dans les années 1960, reflète ce rapprochement surprenant entre la France de cette époque et l'URSS : "Au fond, la France gaulliste était une Union soviétique qui aurait réussi...». pouvoir sous Staline, Paris, La Découverte, 2003. 
mathématique déjà très vivante avant la Révolution. Elles visaient à quantifier les besoins de la population. Puis après 1930, la comptabilité liée au Plan autoritaire stalinien a pris la place de cette statistique sophistiquée. Plusieurs statisticiens ont été fusillés. La stakhanovisme a ainsi été un précurseur brutal des indicateurs de performance du NPM. Après les années 1960, la statistique soviétique était, en Occident, réputée de mauvaise qualité, sinon complètement fausse, car associée aux prévisions et aux réalisations du Plan, et donc sujette à toute sorte de manipulations. Or cette critique ressemble beaucoup à celle des indicateurs du NPM, pour laquelle les chercheurs anglais ${ }^{19}$ parlent de "gaming and cheating " (jouer et frauder). Le point commun à ces deux situations, bien sûr très différentes, est constitué par les effets pervers de la rétroaction des indicateurs sur les acteurs quantifiés.

À l'opposé, l'« État libéral " classique (qui naît à la fin du $\mathrm{XVIII}^{\mathrm{e}}$ siècle) réduit au minimum son intervention. Il prône la libération des forces du marché. Le rêve d'une société « libéralelibertaire " sans État, fondée sur les seuls mécanismes du marché, où les prix intègreraient toute l'information nécessaire, est le symétrique du rêve précédent d'un pur État ingénieur. Les statistiques, si elles existent, visent à rapprocher les marchés réels de ceux de la théorie (information complète et identique pour tous les acteurs), notamment en matière de prix. Cependant, les enquêtes agricoles menées aux États-Unis depuis plus d'un siècle montrent que cette société de marché sans État est une utopie. Elles visent en effet à garantir à tous les fermiers une égale information sur les prévisions de récolte, et à éviter la spéculation des gros acheteurs. Elles impliquent une organisation administrative complexe et un État fédéral bien organisé ${ }^{20}$. Dans ce cas la rétroaction est globale : elle porte sur la construction d'un marché assurant la justice des relations entre offreurs et demandeurs.

19 Christopher Hood, "Control, Bargains, and Cheating: The Politics of Public-Service Reform ", Journal of Public Administration Research and Theory, vol. $12, n^{\circ} 3$, 2002, p. 309-332.

20 Emmanuel Didier, En quoi consiste l'Amérique? Les statistiques, le New Deal et la démocratie, Paris, La Découverte, 2009. 
L' «État providence " cherche à protéger les travailleurs salariés des conséquences de l'extension de la logique marchande au travail lui-même $e^{21}$. Il organise des systèmes de protection contre le chômage, les accidents du travail, la maladie, et pour la famille. Il se constitue dans le sillage de la grande crise sociale et économique de la fin du XIX ${ }^{e}$ siècle. Ses instruments statistiques sont centrés sur le travail salarié : notamment les enquêtes par sondage sur l'emploi, sur les besoins, les revenus et les budgets de famille des travailleurs, ainsi que les indices de prix des consommations de ceux-ci. Les statistiques officielles de ce temps portent sur ces thèmes. Les enquêtes par sondage sur les conditions de vie des familles ouvrières apparaissent dans de contexte. Pour ce faire, le Norvégien Anders Kiaer en $1895^{22}$ et l'Italien Corrado Gini en $1921^{23}$ utilisent la méthode du " choix judicieux " (purposive selection). L'Anglais Arthur Bowley en 1905 formule celle du " choix aléatoire " (random selection), et définit " l'intervalle de confiance " probabiliste. Là encore, la rétroaction est globale, en ce qu'elle concerne l'ensemble de la classe ouvrière, par exemple dans le cas de l'indice des prix à la consommation utilisé pour indexer les salaires, dans le cadre des négociations entre syndicats et patronat. Mais cet indice ne porte que sur les consommations des travailleurs salariés ${ }^{24}$.

L’ État keynésien », pour sa part, a une responsabilité dans le pilotage macroéconomique d'une société dont le caractère marchand n'est cependant pas contesté. Il apparaît dans le contexte de la grande crise des années 1930, et est prédominant entre 1945 et 1975. La comptabilité nationale est son instrument central $^{25}$. Les systèmes statistiques publics sont réorganisés pour

21 Karl Polanyi, The Great Transformation : The Political and Economic Origins of Our Time, Boston, Beacon Hill, 1944.

22 Einar Lie, "The Rise and Fall of Sampling Methods in Norway, 1875-1906 ", Science in Context, $\mathrm{n}^{\circ}$ 3, 2002, p. 385-409.

23 Jean-Guy Prévost, A Total Science: Statistics in Liberal and Fascist Italy, Montréal, McGill Queen's University Press, 2009.

24 Alain Desrosières, " Du travail à la consommation : l'évolution des usages des enquêtes sur le budget des familles ", Journal de la Société française de statistique, $\mathrm{n}^{\text {os }} 1-2,2003$, p. 75-111.

25 François Fourquet, Les Comptes de la puissance. Histoire de la comptabilité 
les besoins de celle-ci. La consommation et l'indice des prix quantifiant l'inflation concernent dorénavant toute la population et non plus les seuls travailleurs salariés. Les modèles macro économétriques orientent des politiques portant sur des agrégats, en confrontant offre et demande globale ${ }^{26}$. La rétroaction globale concerne ces politiques macroéconomiques, et non les acteurs au niveau microéconomique. Dans les deux cas des États Providence et keynésien, les rétroactions résultent souvent d'indexations, notamment sur l'indice des prix ou sur le Produit intérieur brut (exemple des règlements européens).

Enfin l'" État néolibéral " prend appui sur les dynamiques microéconomiques marchandes, en les orientant éventuellement par des systèmes d'incitations et en acceptant les principales hypothèses de la théorie des anticipations rationnelles. Il prend son essor à la suite de la grande crise des années 1970. La rétroaction y prend notamment la forme du benchmarking, c'est-à-dire de l'évaluation, du classement et du palmarès des performances ${ }^{27}$. Les modèles microéconométriques permettent de séparer et d'isoler les « effets propres » de variables ou d'outils de l'action publique sur les performances de ceux-ci, en vue d'améliorer les « variables cibles » de politiques pensées en termes d'incitations (notamment fiscales) et de comportements individuels. L'émulation suscitée entre les outils d'action publique permet de dégager les "meilleures pratiques".

L'évaluation des procédures incitatives résulte d'études sur données individuelles, ou de quasi-expérimentations (ou microsimulations) visant à modéliser les comportements des acteurs, $y$ compris ceux de la puissance publique. Ce point est une différence importante entre l'État néolibéral et les précédents. Il résulte de

nationale et du Plan, Paris, Encres-Recherches, 1980; André Vanoli, Une Histoire de la comptabilité nationale, Paris, La Découverte, 2002, traduction anglaise : A History of National accounting, Amsterdam, IOS Press, 2005.

26 Michel Armatte, La Science économique comme ingénierie, Paris, Presses de l'École des mines, 2010; Michel Armatte et Amy Dahan, "Modèles et Modélisations (1950-2000) : nouvelles pratiques, nouveaux enjeux ", Revue d'histoire des sciences, vol 57, n² 2, 2004, p. 243-303.

27 Isabelle Bruno, À vos marques, prêts... cherchez! La stratégie européenne de Lisbonne, vers un marché de la recherche, Paris, Éditions du Croquant, 2008. 
la théorie des anticipations rationnelles, pour qui les politiques publiques échouent dès lors que les acteurs intègrent, dans les informations orientant leurs comportements, les effets anticipés de ces décisions publiques. Dans cette perspective, aucun acteur, notamment l'État, n'est extérieur au jeu. L'État se démultiplie en plusieurs centres de direction plus ou moins autonomes, ou « agences " gérées comme des quasi-entreprises. Celles-ci sont des acteurs parmi d'autres, relevant des mêmes formes de modélisations que n'importe quel autre acteur microéconomique.

\section{Qualifier, comparer, évaluer, classer : la politique des indicateurs statistiques}

À la différence des activités marchandes, les politiques publiques ne disposent pas de critères comptables tels que la "part de marché » ou la rentabilité, pour juger leur capacité à satisfaire les besoins des usagers, ou leur efficacité. La notion traditionnelle de service public supposait un engagement fort de ses membres, contrôlé par des logiques de subordination hiérarchique, dont l'État français a longtemps constitué un exemple. Mais depuis les années 1980, ce sens civique du service public a été jugé insuffisant pour contrôler efficacement ces activités financées par des ressources publiques. Des indicateurs quantifiés ont été recherchés pour jouer un rôle comparable aux comptabilités analytiques, aux comptes d'exploitation et aux bilans des entreprises marchandes. La comptabilité nationale ne jouait pas ce rôle, puisqu'elle se plaçait au niveau macroéconomique, dans une perspective de politique keynésienne ou de planification indicative, sans entrer dans le détail des actions publiques. Dans cette nouvelle perspective, les indicateurs ne peuvent pas être uniquement monétaires, puisque, le plus souvent, les effets de ces actions (l'école, la santé publique, la sécurité, la diplomatie, la défense) ne sont pas exprimables dans l'espace d'équivalence familier qu'offre la monnaie.

Les efforts dans ce sens menés, tant par l'État français que par l'Union européenne, sont de vastes et tâtonnantes expériences 
pour construire et négocier de nouveaux espaces d'équivalence, en convenant des procédures de quantification des fins et des moyens de l'action, dans des unités diverses, dont la monnaie fait éventuellement partie mais n'est pas la seule. Deux exemples seront évoqués ici, celui de la Loi organique relative aux lois de finances (LOLF), votée à une quasi-unanimité en 2001 par le Parlement français, et celui de la Méthode ouverte de coordination (MOC) utilisée par l'Union européenne ${ }^{28}$. Si les contextes historiques et politiques de ces deux instruments (l'un français, l'autre européen) de gouvernement des politiques publiques, sont différents, ils ont en commun de confier un rôle central à des indicateurs statistiques, c'est à dire à des outils peu évoqués dans les débats publics, alors qu'ils constituent les espaces et les langages mêmes qui délimitent et structurent ces débats.

La LOLF réorganise la présentation du Budget de l'État, selon les objectifs à atteindre et non plus seulement selon les moyens attribués. Ces objectifs doivent être explicités et quantifiés, afin que le Parlement ne se contente plus de voter des dépenses, mais puisse aussi vérifier la réalisation des objectifs et les performances des services. Cette idée de quantification des fins et des moyens de l'action publique va de soi si le Parlement souhaite jouer son rôle constitutionnel de vote et de suivi de l'exécution du Budget. Elle implique cependant un important travail d'objectivation et de mise en équivalence d'activités disparates. Ces activités doivent être parlées, discutées, nommées, qualifiées, comparées, classées, évaluées. L'indicateur à retenir n'est jamais évident. Un ordre social et institutionnel ancien, souvent implicite, est soudain décrit, objectivé. Ceci ne peut se faire, en principe, qu'en impliquant les personnes concernées. Mais, souvent, la notion même d'indicateur quantitatif suscite des réticences. Ces procédures conduisent à " comparer l'incomparable ». Elles appa-

$28 \quad$ Des précédents historiques, certes différents, pourraient être étudiés dans cette perspective : les expériences de planification économique des pays socialistes, ou la "rationalisation des choix budgétaires " $(\mathrm{RCB})$ menée en France dans les années 1970, poursuivie ensuite sous le nom d' «évaluation des politiques publiques ». Dans ces divers cas, des indicateurs quantifiés non monétaires avaient été mis en œuvre. 
raissent parfois absurdes, et ceci d'autant plus que les acteurs sont personnellement plus impliqués dans leurs tâches. Le fait de créer des catégories, en principe pour simplifier le monde et le rendre lisible, tout à la fois le modifie et en fait un autre monde. Les acteurs, changeant de système de référence, ne sont plus les mêmes acteurs, puisque leurs actions sont désormais orientées par ces indicateurs et ces classifications, qui deviennent des critères d'action, et d'évaluation de celle-ci.

La LOLF est supposée permettre au Parlement de mieux connaître et évaluer les actions des services publics, dans la perspective d'un rééquilibrage des pouvoirs législatif et exécutif. Mais le fait que ceci impliquait l'invention et la mise en place d'un grand nombre d'indicateurs quantifiés n'a pas attiré beaucoup l'attention des commentateurs, au moins entre 2001 et 2004. Ceci semblait une question technique, à résoudre par des techniciens. Pourtant, les discussions, de plus en plus précises à partir de 2005, montrent que ce moment de la quantification (au sens d'action de quantifier) est décisif pour la suite des événements. Les effets de rétroaction, s'ils sont vus, le sont comme " effets pervers ", sans être étudiés avec quelque généralité. Ces effets apparaissent au coup par coup. Ils font l'objet de dénonciations ou de plaisanteries. Un exemple : la police et la gendarmerie, responsables de la sécurité routière, ont toutes deux choisi un indicateur portant sur la part de tests d'alcoolémie positifs parmi les tests effectués auprès des automobilistes. Mais la première a souhaité évaluer son action par une augmentation de cette part, tandis que la seconde visait en revanche à la diminuer. Ces deux choix ont chacun leur logique. Ce cas montre ce qu'une sociologie politique de la quantification pourrait traiter pour étudier les effets de la "politique des indicateurs " impliquée par la LOLF, ou, au niveau européen, par la Méthode ouverte de coordination.

Dans d'autres contextes, de tels effets ont déjà été observés. La planification centralisée des pays socialistes a échoué parce qu’il était impossible de fixer des indicateurs fiables de réalisation des objectifs du Plan, en raison des effets pervers induits par ces 
indicateurs, par rétroaction sur le comportement des acteurs. Dans le contexte américain, la mise en place d'une classification des professions de l'hôpital, et l'explicitation formalisée d'activités auparavant implicites, ont transformé celles-ci ${ }^{29}$. Les indicateurs et les classifications sont tout à la fois des contraintes et des ressources qui, par leur existence même, changent le monde.

La Méthode ouverte de coordination (MOC) est utilisée par l'Union européenne pour harmoniser des politiques sociales (emploi, éducation, assistance) ne relevant pas des domaines économiques et monétaires qui sont, eux, explicitement de sa compétence. Un exemple en a été la Stratégie européenne pour l'emploi (SEE) proposée en 1997. Le principe en est que, de façon intergouvernementale, les États se fixent des objectifs communs, eux mêmes exprimés par des indicateurs quantifiés, par rapport auxquels les États seront ensuite évalués et classés, comme dans un palmarès. Les résultats de ce benchmarking sont en principe indicatifs, mais le simple fait d'être publiés constituent un stimulant pour orienter les politiques nationales dans les directions indiquées lors des Sommets ${ }^{30}$. Un objectif de taux d'emploi de $70 \%$ a ainsi été fixé. La LOLF et la MOC confèrent un rôle clé aux indicateurs statistiques, l'une pour le suivi du Budget de l'État, l'autre pour le pilotage indirect des politiques sociales européennes.

La façon dont les États de l'Union conviennent des méthodes de cette quantification est essentielle, bien que mal connue. Techniquement, ce travail est divisé en deux parties. Les autorités politiques décident du choix des indicateurs et les définissent de façon succincte avec des mots. Puis ils transmettent une commande de quantification aux statisticiens d'Eurostat (l'Office

$\overline{29}$ Geof Bowker et Susan Leigh Star, Sorting Things out. Classification and Its Consequences, Cambridge (MA), MIT Press, 1999.

30 Renaud Dehousse, "La Méthode ouverte de coordination. Quand l'instrument tient lieu de politique ", dans Pierre Lascoumes et Patrick Le Galès (dir.), Gouverner par les instruments, Paris, Presses de Sciences Po, 2004, p. 331-356; Robert Salais, "La Politique des indicateurs. Du taux de chômage au taux d'emploi dans la stratégie européenne pour l'emploi (SEE) ", dans Bénédicte Zimmermann (dir.), Action publique et sciences sociales, Paris, MSH, 2004, p. 287-331. 
statistique de l'Union européenne) et des Instituts nationaux de statistique (INS). La phase " convenir " est donc elle-même partagée, puisque les responsables politiques laissent aux statisticiens le soin de régler les "détails ", comme par exemple les définitions précises des notions de taux d'emploi ${ }^{31}$, de revenu disponible d'un ménage ${ }^{32}$, de sans abri ${ }^{33}$ Ces études montrent que les statisticiens ne peuvent éviter, compte tenu des différences institutionnelles entre les pays, de laisser dans le flou certaines spécifications, parfois importantes, des procédures de mesures, et ne peuvent pas les harmoniser complètement. Cette méthode est dite " ouverte » car elle n'est pas impérative, et laisse les États libres de l'adapter à leurs particularités institutionnelles, notamment en choisissant comme sources des enquêtes directes ou des registres administratifs ${ }^{34}$.

Ces indicateurs ont un caractère flou, non exhaustivement défini. Ceci leur permet de servir dans plusieurs univers, qui auparavant s'ignoraient et peuvent désormais se comparer, comme un langage commun. Le langage naturel a des propriétés analogues : c'est parce que les locuteurs ne passent pas leur temps à expliciter le sens et le contenu des mots prononcés que la communication est possible. Les objets de la statistique publique, le taux de chômage, l'indice des prix, le PIB, sont dans le même cas. Une explicitation de leur mode de construction et de leur contenu risquerait d'affaiblir leur efficacité argumentative, non seulement parce qu'elle dévoilerait des conventions ou des approximations non soupçonnées par l'utilisateur, mais tout bonnement pour des raisons d'économie du cours des échanges, des débats, des démonstrations dans lesquels ces arguments statistiques trouvent place. Ceci reste en général implicite, sauf en cas de controverse. Cependant, cette idée de flou ne peut que

\footnotetext{
$31 \quad$ Ibid.

32 Delphine Nivière, "Négocier une statistique européenne: le cas de la pauvreté ", Genèses, n 58, 2005, p. 28-47.

33 Cécile Brousse, "Définir et compter les sans-abri en Europe : enjeux et controverses ", Genèses, n 58, 2005, p. 48-71.

34 Alain Desrosières, "Décrire l'État ou explorer la société: les deux sources de la statistique publique ", Genèses, n 58, 2005, p. 4-27.
} 
choquer, à juste titre, des professionnels soucieux de définir et de standardiser leurs objets. Ils sont pris entre deux exigences contradictoires. D'une part, ils souhaitent, en bons ingénieurs, spécifier leurs procédures. Mais d'autre part, les négociations les incitent à tolérer des compromis sans lesquels les indicateurs demandés seraient impossibles à fournir. L'équilibre que, de fait, ils cherchent à maintenir entre ces deux exigences, est peu explicité et formalisé 35 .

\section{Pour une sociologie de la rétroaction : le cas de la comptabilité}

"Quand une mesure devient une cible, elle cesse d'être une bonne mesure " ("Loi de Goodhart ", citée par Sheila Bird ${ }^{36}$ )

Si la question de la rétroaction est peu familière au statisticien, elle est en revanche très présente dans les réflexions des comptables, et maintenant, par extension, dans les débats sur l'usage des indicateurs quantifiés de gestion. Nous en mentionnerons ici quelques exemples, issus de traditions théoriques très différents, depuis la microéconomie néo-classique jusqu’à la sociologie et la science politique inspirées de Michel Foucault. La "Loi de Goodhart " en est un symbole. Charles Goodhart était un conseiller de la Banque d'Angleterre. Sa « loi », formulée en 1975, devint célèbre quand le gouvernement de Margaret Thatcher entreprit de piloter sa politique monétariste sur la base de " cibles " pour le montant de la masse monétaire. Elle était déjà implicite dans l'idée $\mathrm{d}$ ' " anticipation rationnelle " et dans la critique de Lucas contre les politiques keynésiennes ${ }^{37}$. Sa première formulation était : "Toute régularité statistique observée devient fausse si une pression est exercée sur elle à des fins de

35 Cette ambiguïté est cependant perceptible à propos des méta-données (les données sur les données). Elles sont demandées et fournies, mais donner trop de détails peut introduire un doute insidieux, non souhaité. L'argument statistique est plus efficace s'il est invoqué dans sa nudité, sans notes de bas de page.

36 Sheila Bird, "Editorial: Performance monitoring in the public services ", Journal of the Royal Statistical Society, A, $\mathrm{n}^{\circ} 167$, Part 3, p. 381-383.

37 Alec Chrystal et Paul Mizen, "Goodhart's Law: Its Origin, Meaning and Implications for Monetary Policy ", Festschrift in Honour of Charles Goodhart, $\mathrm{n}^{\mathrm{os}} 15-16$, November 2001, Bank of England. 
contrôle ". Elle fut étendue ensuite à tout indicateur utilisé comme cible. La formule citée ci-dessus est mentionnée le 15 juin 2004 dans un éditorial du Journal of the Royal Statistical Society intitulé : "Performance monitoring in the public services ». L'idée d' "incitation perverse », classique dans la littérature du management, en résulte. La question existentielle est " Une mesure des affaires humaines peut elle être complètement indépendante de ses usages? ». L'ethos de neutralité métrologique du statisticien est mal à l'aise avec cette question. C'est pourquoi l'État néolibéral, basé sur des indicateurs de performance, a des difficultés avec ses statistiques, comme en avait naguère la statistique soviétique.

La comptabilité d'entreprise est un domaine pour lequel une sociologie de la rétroaction peut se déployer, compte tenu de la diversité de ses usages et de la malléabilité de ses normes, surtout dans le monde anglo-saxon, où on parle ironiquement de creative accounting (comptabilité créative), de window dressing (arranger la vitrine), ou de cooking the book (faire cuire le livre). Ainsi par exemple, pour " valoriser " les actifs du bilan, trois conventions peuvent être employées, correspondant à trois logique d'usage. 1) Le coût d'origine (ou valeur historique) est utilisé par le gestionnaire qui cherche à répartir des annualités d'amortissement. 2) La valeur de revente préoccupe le créancier de l'entreprise, qui se demande ce que vaut encore son actif. Enfin 3) la somme des revenus futurs actualisés intéresse l'investisseur, qui répartit ses disponibilités financières ${ }^{38}$. La dernière convention (dite Fair Value), a été retenue par l'Union européenne, au détriment de la première, par les nouvelles normes comptables internationales ${ }^{39}$, les IFRS (International Financial Reporting Standards) ${ }^{40}$. Une

38 Selon la théorie néo-classique dite " des marchés efficients », les conventions 2 (valeur de revente) et 3 (somme des revenus futurs actualisés) sont équivalentes.

39 Cette convention de Fair value rend la valeur des actifs très volatile et incertaine en cas de crise. Ceci a été une des causes de la propagation de la crise de 2008.

40 Ėve Chiapello et Karim Medjad, «Une Privatisation inédite de la norme : le cas de la politique comptable europénne ", Sociologie du travail, n 49, 2007, p. 46-64. 
diversité comparable existe pour les différentes manières de calculer le profit de l'entreprise, selon les objectifs de ce calcul. La forme active du verbe "valoriser " utilisé par les comptables, est significative d'une démarche implicitement plus constructiviste que réaliste. Là où l'économiste débat des " fondements de la valeur ", le comptable "valorise ", c'est-à-dire fabrique une valeur, selon des conventions. À l'intérieur même des règles et des conventions légales d'établissements des comptes, les entreprises disposent de degrés de liberté leur permettant de faire apparaître un bénéfice plus ou moins élevé, selon qu'elles sont soucieuses du message transmis à leurs actionnaires, à d'éventuels repreneurs, à l'État, ou à d'autres acteurs de l'économie ${ }^{41}$.

La flexibilité des normes comptables a été à l'origine d'une étrange mais influente "positive accounting theory" (PAT) ${ }^{42}$. Cette branche de la recherche académique en comptabilité cherche à expliquer et prédire les pratiques comptables réelles. Elle s'oppose à la comptabilité normative. Celle-ci vise à définir et prescrire des standards théoriques optimaux en tant que « reflets de la réalité ", en répondant à des questions comme : "Qu'est ce qu'un revenu? ", "Qu'est ce qu'un actif? », à partir de raisonnements déductifs, sans se référer à ce que les comptables font en réalité. En revanche, les "positivistes ", inspirés notamment par la "théorie de l'agence ", affirment avoir à analyser les choix et les pratiques réels des comptables, avant de dire ce que ceux-ci devraient faire. Ils ne s'intéressent pas à ce que les comptables disent en termes métrologiques, mais à leurs comportements stratégiques révélés par leur pratique. Pour cela ils mobilisent des

$41 \quad$ Sur toutes ces questions, l'Encyclopédie de comptabilité, contrôle de gestion et audit (2009), éditée par Bernard Colasse, est une somme très utile.

42 Ross Watts et Jerold Zimmerman, "Towards a Positive Theory of the Determination of Accounting standards ", The Accounting Review, vol. 53, $\mathrm{n}^{\circ} 1,1978$, p. 112-134. Ce paragraphe sur la positive accounting theory doit beaucoup à un travail mené en commun avec Ève Chiapello, professeure à l'École des Hautes études commerciales (HEC) de Paris. Voir Ėve Chiapello et Alain Desrosières, "La Quantification de l'économie et la recherche en sciences sociales : paradoxes, contradictions et omissions. Le cas exemplaire de la Positive Accounting Theory ", dans François Eymard-Duvernay (dir.), L'Économie des conventions. Méthodes et résultats, Tome 1, Débats, Paris, La Découverte, 2006, p. 297-310. 
corrélations statistiques et des outils économétriques pointus pour modéliser les pratiques comptables associées à des buts stratégiques. Ainsi l'objectif métrologique de la quantification comptable semble disparaître, au profit de l'analyse sophistiquée de l'effet de rétroaction Là est le paradoxe : une méthode " positive » est mise au service d'une conception complètement relativiste de la quantification de l'économie, très éloignée d'une idée de "valeur fondamentale ». La crise de 2008 a fourni des exemples de conséquences de ce genre de théorie, notamment avec l'usage de la désormais célèbre Fair value.

Une toute autre sociologie de la rétroaction est à l'œuvre dans les travaux de chercheurs anglais de diverses disciplines, regroupés autour de Anthony Hopwood dans la revue Accounting, Organizations, and Society (AOS), ou de Christopher Hood à Oxford. La Grande-Bretagne a connu la vague du néolibéralisme bien avant la France, dés les années 1980, après l'élection de Margaret Thatcher ${ }^{43}$. Ceci explique que la question de la rétroaction et, plus généralement celle des traits spécifiques de la gouvernementalité néolibérale, y ont été posées dès ce moment, notamment par ces chercheurs ${ }^{44}$. La Revue $A O S$ se pose, à propos de la rétroaction, des questions analogues à celles de la PAT, mais elle les traite de façon radicalement différente de celle-ci. Elle resitue la comptabilité dans son contexte historique, sociologique et politique, alors que la PAT recourt aux seuls outils de la

43 Patrick Le Galès, "Contrôle et surveillance. La restructuration de l'État en Grande-Bretagne ", dans Pierre Lascoumes et Patrick Le Galès (dir.), Gouverner par les instruments, Paris, Presses de Sciences Po, 2004, p. 237-271.

44 Peter Miller et Timothy O'Leary, "Accounting and the Construction of the Governable Person ", Accounting, Organization, and Society, vol. 12, $\mathrm{n}^{\circ} 3$, 1987, p. 235-265; Peter Miller, "Accounting and Objectivity: The Invention of Calculating Selves and Calculable Spaces ", Annals of Scholarship, vol. 9, $\mathrm{n}^{\text {os }}$ 1-2, 1992, p. 61-86; Anthony Hopwood et Peter Miller (dir.), Acounting as Social and Institutional Practice, op. cit.; Christopher Hood, "Contemporary Public Management»: A New Paradigm? ", op. cit.; Christopher Hood, "Control, Bargains, and Cheating: The Politics of Public-Service Reform ", op. cit.; Michael Power, The Audit Society. Rituals of Verification, op. cit.; Michael Power, "Counting, Control, and Calculation: Reflections on Measuring and Management ", Human Relations, vol. 57, n 6, 2004, p. 765783. 
microéconomie, de la théorie de Hopwood l'agence et de l'économétrie. AOS mobilise une vaste palette de sciences sociales ${ }^{45}$. Dès 1991, ces chercheurs se référaient aux écrits de Michel Foucault sur la gouvernementalité, pour analyser la gestion néolibérale ${ }^{46}$, bien avant que ne soient publiés en français (en 2004) ses cours sur ce sujet, et que cette lecture ne se généralise chez des chercheurs en gestion ${ }^{47}$ et des sociologues ${ }^{48}$. Ceci résulte de ce que le New Public Management avait été diffusé en GrandeBretagne dès les années $1980^{49}$.

Ces chercheurs anglais retenaient de Foucault les idées d'action à distance, de conduite des conduites et d'entrepreneur de soi-même. Le thème de l'accountability y est central. Ce mot, difficile à traduire en français, reflète à la fois la responsabilité et l'obligation de rendre compte et d'évaluer résultats et performances. Eric Pezet $^{50}$ exprime bien cette combinaison à la fois morale et technique : "L'entrée en comptabilité des individus ne les rend pas seulement responsables, ils deviennent comptables de leur comportement à partir d'échelles de mesure qui sont données par les services de gestion des ressources humaines et par les managers ».

45 L'histoire de la ligne éditoriale de la Revue AOS depuis 1975, a été analysée à travers les éditoriaux de son fondateur, Anthony Hopwood, par Stéphane Lefrancq, "Recherche et action : la comptabilité dans son contexte. Une étude de la politique éditoriale d'Accounting, Organizations and Society ", Comptabilité-Contrôle-Audit, numéro thématique, juin 2004, p. 297-315.

46 Graham Burchell, Colin Gordon et Peter Miller (dir.), The Foucault Effect. Studies on Governmentality, Chicago, Universty of Chicago Press, 1991.

47 Eric Pezet (dir.), Management et conduite de soi. Enquête sur les ascèses de la performance, Paris, Vuibert, 2007.

48 Pierre Dardot et Christian Laval, La Nouvelle Raison du monde. Essai sur la société néolibérale, Paris, La Découverte, 2009.

49 Avec d'autres catégories analytiques et un autre vocabulaire, (ils parlaient de capitalisme et non de néolibéralisme), Luc Boltanski et Ève Chiapello, Le Nouvel Esprit du capitalisme, Paris, Gallimard, 1999, abordaient déjà la question des changements radicaux des modes de gestion du NPM. Ils analysaient un autre type de rétroaction, celle de la critique sous ses diverses formes, sur l'ensemble du capitalisme. De ce point de vue, les travaux sur les controverses et critiques à propos des normes IFRS, et leurs rôles dans le capitalisme financier, sont précieux pour notre projet de sociologie de la rétroaction. Voir Michel Capron (dir.), Les Normes comptables internationales, instruments du capitalisme financier, Paris, La Découverte, 2005.

50 Eric Pezet, op. cit. 
À la gestion par commandement direct se substitue une gestion indirecte, fondée sur la conduite des conduites des autres, et l'intériorisation des contraintes par le sujet, devenu ainsi « entrepreneur de lui-même $"^{51}$. Dès lors, la rétroaction des indicateurs quantitatifs atteint l'individu dans tous les moments de sa vie. Ce mode de gestion peut avoir, on le sait, des répercussions graves sur l'équilibre psychique des individus, les conduisant parfois jusqu'au suicide.

Peter Miller introduisit, dans son texte de $1992^{52}$, deux notions bien reliées à notre idée de rétroaction : les calculating selves (sois calculateurs) et les calculable spaces (espaces calculables). Ainsi la quantification et le calcul circulent en va-et-vient entre l'individu, qui s'évalue et se calcule lui-même (self), et son environnement (space), qui lui dicte les façons de calculer, en l'enserrant dans un carcan paré des atours de la liberté. Miller indique cinq traits spécifiques de cette façon d'utiliser les indicateurs quantifiés de performance.

1. Les "sois calculateurs" et les "espaces calculables" permettent d'agir sur les actions des autres. Mais les indicateurs sont " mal reliés entre eux " (loosely linked to each other). [Ce trait est caractéristique de cette façon de mobiliser les statistiques pour le NPM, sous forme de "batterie d'indicateurs", accumulés et peu cohérents, par différence avec les agrégats de la comptabilité nationale, fortement reliés entre eux par des contraintes d'équilibre comptable et des modèles économiques].

2. L'expertise calculatoire remplace l'autorité professionnelle. Le politique et le moral deviennent du factuel et du calculable. Le débat social contradictoire est affaibli au profit de cette ingénierie qui se présente comme objective et neutre.

3. Cela ne marche pas, mais ce n'est pas grave. Les indicateurs quantitatifs sont critiqués, mais cela ne discrédite pas du tout le système, qui est toujours prêt à se transformer. Ils

\footnotetext{
51 Le nouveau statut juridique français d'auto-entrepreneur en est une saisissante illustration.

52 Peter Miller, «Accounting and Objectivity: The Invention of Calculating Selves and Calculable Spaces", op. cit.
} 
sont supposés " être améliorés en permanence, au vu de l'expérience ». [Exemple : les palmarès des Universités depuis celui de Shangai].

4. Il y a des résistances, de la part de professionnels dépossédés de leurs spécificités et de leurs territoires propres par ces expertises calculatoires, venues d'ailleurs et indépendantes des terrains où elles sont appliquées. [Exemple : les mouvements de protestation de l'hiver 2009 en France, dans les Universités, les écoles, les hôpitaux, ou la psychiatrie, relevaient précisément de ce type de résistance].

5. Le futur devient connaissable, calculable et contrôlable, moyennant le choix et l'usage d'un taux d'actualisation, convention d'équivalence entre le présent et le futur.

\section{Les conditions de félicité de l'argument statistique}

Ces analyses semblent, du moins pour le moment, éloignées des questions que se posent les statisticiens, dont la pratique et l'ethos professionnels sont différents de ceux des comptables. L'abondante littérature sur la comptabilité et la gestion, anglophone ou francophone, est largement ignorée des statisticiens et des économistes, bien que les sources de leurs statistiques en soient souvent issues. Ou plutôt, si ils soupçonnent que la comptabilité ne reflète pas aussi adéquatement qu'ils le souhaiteraient, la " réalité ", c'est pour le déplorer, comme le montre le livre célèbre, déjà ancien, d'Oskar Morgenstern (1944/1963) ${ }^{53}$. Supposer que l'ethos des statisticiens est différent de celui des comptables n'implique pas qu'ils soient plus honnêtes, mais que leur insertion sociale et administrative, et leurs raisons d'agir, ne sont pas les mêmes. Ceci peut se traduire concrètement par des " codes de déontologie " et des " chartes de bonnes pratiques ", mais, plus profondément, cet ethos implique une culture professionnelle spécifique. Celle-ci combine (idéalement), de façon origi-

53 Oskar Morgenstern, On the Accuracy of Economic Observations, Princeton, op. cit. Le titre de sa traduction française chez Dunod en 1950 était, significativement : L'Illusion statistique. Précision et incertitude des données économiques. 
nale, la posture scientifique et le sens de l'État ${ }^{54}$.

Il est difficile de caractériser une statistique publique qui serait spécifique de l'État néolibéral, comme on avait pu le faire pour les formes d'État antérieures. Pourtant, plusieurs développements récents montrent que les phénomènes de rétroaction n'en sont pas absents. Ainsi les statisticiens ont été sollicités pour définir et quantifier les indicateurs de la Méthode ouverte de coordination (MOC) de l'Union européenne, fondée sur le benchmarking des performances de États, un outil typique de cette nouvelle gouvernementalité. L'application des critères du traité de Maastricht (déficit des finances publiques, dette des administrations) a posé des problèmes de rétroaction des indicateurs quantitatifs, impliquant ce que les comptables appellent du window dressing, comme le cas de la Grèce l'a montré.

Autre exemple : un âpre conflit sur les statistiques du chômage a eu lieu en France en 2007. Les fichiers de l'Agence pour l'emploi subissaient des manipulations flagrantes (bien décrites par Florence Aubenas dans le texte cité en exergue). Celles-ci rendaient fragile la série statistique conjoncturelle du chômage, très attendue par les responsables politiques et par la presse. Les statisticiens ont combattu ces interventions, profondément étrangères à leur ethos professionnel ${ }^{55}$. Il est possible que les analyses de la rétroaction produites par les sociologues de la comptabilité et de la gestion deviennent pertinentes pour les statisticiens, même si, aujourd'hui encore, leur nécessaire indépendance est officiellement affirmée par leurs institutions. Dans ce cas, les batailles (mentionnées ci-dessus : point 4 de Peter Miller) autour de la perte de spécificité et d'autonomie professionnelles, concerneraient les quantificateurs eux-mêmes. Les statisticiens de certains pays sont déjà dans cette situation.

\footnotetext{
54 Ceci implique une revendication récurrente d'indépendance par rapport aux autorités politiques. Cette indépendance est désormais inscrite dans le « Code des bonnes pratiques de la statistique européenne ", adopté en 2005 à la suite de la première "crise grecque ", ce qui vise à freiner, sinon interdire, les rétroactions (http://www.cnis.fr/agenda/DPR/Dpr_0291.pdf).

55 Une bonne illustration de cette exigence est fournie par un colloque syndical organisé en 2007 sous le titre : États généraux des chiffres du chômage et de la précarité. (http://cgtinsee.free.fr/dossiers/chomage/etat-actes.pdf).
} 
Pourquoi mettre en avant cette notion étrange de rétroaction? Toute quantification n'a-t-elle pas, en fin de compte, un usage et des effets? Sinon, pourquoi serait fait le travail, toujours coûteux, de construction de conventions puis de mesure? Cela est bien vrai. Cette idée n'a émergé qu'en raison de la division sociale des activités de production et d'usage des connaissances, entre professions ou entre disciplines académiques, dotées de cultures professionnelles différentes. L'autonomisation d'un métier de statisticien, justifiée par l'importance de l'usage des statistiques comme langage commun spécifique d'une forme de gouvernement, a eu des conséquences contradictoires. D'une part, l'efficacité de l'argument statistique implique que les effets éventuels de rétroaction soient méconnus, sinon déniés, au moment de son énonciation. En paraphrasant les linguistes, on peut parler de condition de félicité de cet argument. Mais d'autre part, ces effets ayant bel et bien lieu, comment le statisticien peut il en prendre conscience et les assumer? La théorie des actes de langage ${ }^{56}$ a, de longue date, mis en scène la distinction entre énoncés dits constatifs et performatifs, sous le titre How to do things with words?, que l'on peut transposer ici en : How to do things with numbers? La distinction constatif-performatif, puis la notion même de performativité, ont été discutées et critiquées dans une abondante littérature, d'abord en linguistique, puis en sociologie et en économie ${ }^{57}$. Notre notion de rétroaction, bien que différente, peut y être rattachée. Les critiques de la quantification évoquées en introduction peuvent être relues de ce point de vue, en insistant sur les causes et les effets de la division du travail de la production et de la circulation des énoncés quantifiés.

Deux critiques de la quantification, portées par des acteurs différents, ont surgi depuis les années 1990. D’une part les conventions d'équivalence impliquées par le NPM pour «évaluer

\footnotetext{
56 John Langshaw Austin, How to do Things with Words, Oxford, Clarendon, 1962, traduction française, Quand dire, c'est faire, Paris, Seuil, 1970.

57 Michel Callon, "What Does It Mean to Say that Economics Is Performative? ", dans Donald MacKenzie, Fabian Muniesa et Lucia Siu (dir.), Do Economists Make Markets? On the Performativity of Economics, Princeton, Princeton University Press, 2007, p. 311-357.
} 
les performances ", sont vivement contestées, on l'a vu, par de nombreux professionnels. D'autre part, à un niveau plus macroéconomique, la "mesure de la richesse " d'une nation au moyen du Produit intérieur brut (PIB) est contestée, et une autre quantification est réclamée, sans d'ailleurs que soient précisées les nouvelles conventions d'équivalence nécessaires pour ce faire. Ces deux critiques semblent sans rapport, énoncées dans des contextes très éloignés. Pourquoi les rapprocher? Les deux ne sont intelligibles que si on les intègre, dans chaque cas, au " triplet " formé de : 1) une façon de penser la société, 2) des façons d'agir sur elle, et 3) la quantification adéquate ${ }^{58}$. L'explicitation de ce triplet éclaire non seulement une forme de gouvernementalité, mais aussi les modalités de sa critique.

La critique des indicateurs de performance du NPM repose sur le fait de mettre en équivalence (ou commensurer), d'évaluer et classer des acteurs et des actions jugés incommensurables, et d'agir sur eux par des mécanismes incitatifs. Les indicateurs visent à outiller ce benchmarking ${ }^{59}$. La commensuration est un acte social qui transforme le monde, notamment en le rendant calculable et classable selon des rankings (palmarès) ${ }^{60}$. Cette critique de la commensuration porte notamment sur les effets pervers des rétroactions. Elle ne prend pas appui sur une autre forme de quantification. Elle revendique au contraire un respect de l'unicité et de la spécificité de chaque acteur et chaque action. Ce geste de résistance a toute sa place dans les débats sur le rôle social du nombre.

\footnotetext{
58 Alain Desrosières, "Historiciser l'action publique : l'État, le marché et les statistiques", op. cit.

59 Isabelle Bruno, À vos marques, prêts... cherchez! La stratégie européenne de Lisbonne, vers un marché de la recherche, op. cit.

60 Ces deux aspects ont été analysés par deux articles de Wendy Espeland aux titres significatifs : Wendy Espeland et Mitchell Stevens, "Commensuration as a Social Process ", Annual Review of Sociology, n² 24, 1998, p. 313-343; Wendy Espeland et Michael Sauder, "Rankings and Reactivity: How Public Measures Recreate Social Worlds ", American Journal of Sociology, n 113, 2007, p. 1-40. On retrouve une critique analogue dans l'ouvrage d'Alain Supiot, L'Esprit de Philadelphie : la justice sociale face au marché total, Paris, Seuil, 2010, sur la disparition de "L'Esprit de Philadelphie ", caractéristique de la philosophie progressiste des trois décennies qui ont suivi 1945.
} 
L'autre critique, symbolisée par le débat sur " le nécessaire dépassement du PIB », se situe à l'opposé, dans une perspective de commensuration généralisée, puisque l'indicateur souhaité viserait à agréger, ou au moins "prendre en compte ", le maximum d'éléments supposés méconnus du PIB classique : l'environnement (climat, biodiversité), le travail non payé, les inégalités, etc., selon le principe : "Au delà du PIB : réconcilier ce qui compte et ce que l'on compte ${ }^{61} »$. Ce projet se situe dès lors dans une perspective implicite (et encore peu formalisée) d'une sorte d'État " post-providence " et " post-keynésien ", accordant une grande place à des statistiques d'un nouveau type, encore à imaginer ${ }^{62}$. Cet État donnerait une grande place notamment à la protection de la nature, et à un dépassement du capitalisme prédateur et inégalitaire. Les effets de rétroaction pourraient y être de type macrosociaux, comme dans l'État keynésien, ou de type incitatif, comme dans la Méthode ouverte de coordination européenne.

La tension apparente entre ces deux critiques, l'une hostile à toute commensuration, et la seconde appelant une commensuration adéquate à une autre gouvernementalité, peut être interprétée en termes de philosophie morale, comme une opposition entre un principe déontologique, et un principe téléologique, tels qu'ils sont formulés à propos des questions d'allocation des ressources rares en économie de la santé ${ }^{63}$. Selon l'un (déontologique), chaque personne a une valeur unique, incommensurable à toute autre. On ne peut mettre en balance la vie d'un vieillard

\footnotetext{
61 Isabelle Cassiers et Géraldine Thiry, "Au delà du PIB : réconcilier ce qui compte et ce que l'on compte ", Regards économiques, $\mathrm{n}^{\circ}$ 75, 2009, Institut de Recherches Economiques et Sociales (IRES), en ligne : http://sites. uclouvain.be/econ/Regards/Archives/RE075.pdf.

62 Jean Gadrey et Florence Jany-Catrice, Les Nouveaux Indicateurs de richesse, op. cit., font explicitement la comparaison entre la période actuelle et celle des années 1950, qui avait vu naitre en France la comptabilité nationale et le projet politique keynésien et planificateur qui allait avec, décrit de façon vivante par François Fourquet, Les Comptes de la puissance. Histoire de la comptabilité nationale et du Plan, op. cit.

63 Anne Fagot-Largeault, « Réflexions sur la notion de qualité de la vie », Archives de philosophie du droit, tome 36, volume " Droit et Science », 1991, p. 135153.
} 
et celle d'un jeune homme. Selon l'autre (téléologique, ou utilitariste), il existe un bien commun supérieur aux individus, justifiant que la collectivité fasse des arbitrages, notamment pour l'affectation des ressources économiques limitées à des besoins de santé publique potentiellement illimités. Si l'économicisme du néolibéralisme pousse irrésistiblement vers le second principe, dont la justification est évidente, la pleine légitimité du premier doit sans cesse être réaffirmée.

Comment résoudre la contradiction entre l'ethos du statisticien et la prise en compte des rétroactions, même quand celles-ci lui apparaissent seulement comme de fâcheux obstacles à sa mission, qu'il pense être de " fournir des reflets non biaisés de la réalité "? Il n'est pas possible d'isoler un moment de la mesure, qui serait indépendant de ses usages, et notamment des conventions qui sont la première étape de la quantification. Il faudrait désenclaver la formation des statisticiens, en la complétant par des éléments d'histoire, de sciences politiques, et de sociologie de la statistique, de l'économétrie, des probabilités, de la comptabilité et de la gestion. Ce programme, inspiré des acquis des Sciences Studies ${ }^{64}$, pourrait faciliter la prise en compte des outils quantitatifs dans les débats sociaux, sans verser ni dans le rejet a priori, ni dans le respect inconditionnel et naïf devant des « faits incontestables parce que quantifiés ".

$\overline{64}$ Dominique Pestre, Introduction aux Science Studies, Paris, La Découverte / Repères, 2006. 


\section{Bibliographie}

Armatte, Michel, La Science économique comme ingénierie, Paris, Presses de l'École des mines, 2010.

Armatte, Michel et Amy Dahan, « Modèles et Modélisations (1950-2000) : nouvelles pratiques, nouveaux enjeux ", Revue d'Histoire des Sciences, vol. 57, no 2, 2004, p. 243-303.

Aubenas, Florence, Le Quai de Ouistreham, Paris, L'Olivier, 2010.

Austin, John Langshaw, How to do Things with Words, Oxford, Clarendon, 1962, traduction française: Quand dire, c'est faire, Paris, Seuil, 1970.

Bird, Sheila, "Editorial: Performance Monitoring in the Public Services ", Journal of the Royal Statistical Society, A , n 167, Part 3, p. 381-383.

Blum, Alain et Martine Mespoulet, L'Anarchie bureaucratique. Statistique et pouvoir sous Staline, Paris, La Découverte, 2003.

Boltanski, Luc et Ève Chiapello, Le Nouvel Esprit du capitalisme, Paris, Gallimard, 1999.

Boskin, Michael et al., Toward a More Accurate Measure of the Cost of Living: Final Report to the Senate Finance Committee, U.S. Government Printing Office, Washington (DC), 1996.

Bowker, Geof et Susan Leigh Star, Sorting Things Out. Classification and Its Consequences, Cambridge (MA), MIT Press, 1999.

Brousse, Cécile, "Définir et compter les sans-abri en Europe : enjeux et controverses ", Genèses, n 58, 2005, p. 48-71.

Bruno, Isabelle, À vos marques, prêts... cherchez! La stratégie européenne de Lisbonne, vers un marché de la recherche, Paris, Éditions du Croquant, 2008.

Burchell, Graham, Colin Gordon et Peter Miller (dir.), The Foucault Effect. Studies on Governmentality, Chicago, Universty of Chicago Press, 1991.

Caldwell, Bruce, "Hayek and Socialism ", Journal of Economic Literature, $n^{\circ}$ XXXV, 1997, p. 1856-1890.

Callon, Michel, "What Does It Mean to Say that Economics Is Performative? ", dans Donald MacKenzie, Fabian Muniesa et Lucia Siu (dir.), Do Economists Make Markets? On the Performativity of Economics, Princeton, Princeton University Press, 2007, p. 311-357.

Capron, Michel (dir.), Les normes comptables internationales, instruments $d u$ capitalisme financier, Paris, La Découverte, 2005.

Cassiers, Isabelle et Géraldine Thiry, "Au delà du PIB : réconcilier ce qui compte et ce que l'on compte ", Regards économiques, $\mathrm{n}^{\circ}$ 75, 2009, Institut de Recherches Économiques et Sociales (IRES). En ligne : 
http://sites.uclouvain.be/econ/Regards/Archives/RE075.pdf.

Chiapello, Ėve et Alain Desrosières, «La Quantification de l'économie et la recherche en sciences sociales : paradoxes, contradictions et omissions. Le cas exemplaire de la Positive Accounting Theory ", dans François Eymard-Duvernay (dir.), L'Économie des conventions. Méthodes et résultats, Tome 1, Débats, Paris, La Découverte, 2006, p. 297-310.

Chiapello, Ėve et Karim Medjad, « Une Privatisation inédite de la norme : le cas de la politique comptable europénne ", Sociologie du travail, $\mathrm{n}^{\circ}$ 49, 2007, p. 46-64.

Chrystal, Alec et Paul Mizen, "Goodhart's Law: Its Origin, Meaning and Implications for Monetary Policy ", Festschrift in Honour of Charles Goodhart, nos 15-16, November 2001, Bank of England.

Colasse, Bernard (dir.), Encyclopédie de comptabilité, contrôle de gestion et audit, Paris, Economica, 2009.

Dahan, Amy et Dominique Pestre (dir.), Les Sciences pour la guerre, 19401960, Paris, Éditions de l'EHESS, 2004.

Dardot, Pierre et Christian Laval, La Nouvelle Raison du monde. Essai sur la société néolibérale, Paris, La Découverte, 2009.

Daston, Lorraine, "Objectivity and the Escape from Perspective ", Social Studies of Science, vol. 22, no 4, 1992, p. 597-618.

Dehousse, Renaud, "La Méthode ouverte de coordination. Quand l'instrument tient lieu de politique ", dans Pierre Lascoumes et Patrick Le Galès (dir.), Gouverner par les instruments, Paris, Presses de Sciences Po, 2004, p. 331-356.

Desrosières, Alain, « Décrire l'État ou explorer la société: les deux sources de la statistique publique ", Genèses, n 58, 2005, p. 4-27.

Desrosières, Alain, "Du travail à la consommation : l'évolution des usages des enquêtes sur le budget des familles ", Journal de la Société française de statistique, $\mathrm{n}^{\text {os }} 1-2,2003$, p. 75-111.

Desrosières, Alain, Gouverner par les nombres, Paris, Presses de l'École des mines, 2008.

Desrosières, Alain, « Historiciser l'action publique : l'État, le marché et les statistiques ", dans Pascale Laborier et Dany Trom (dir.), Historicités de l'action publique, Paris, Presses universitaires de France, 2003, p. 207-221.

Desrosières, Alain, Pour une sociologie historique de la quantification, Paris, Presses de l'École des mines, 2008.

Didier, Emmanuel, En quoi consiste l'Amérique? Les statistiques, le New Deal et la démocratie, Paris, La Découverte, 2009.

Espeland, Wendy et Mitchell Stevens, "Commensuration as a Social 
Process ", Annual Review of Sociology, n² 24, 1998, p. 313-343.

Espeland, Wendy et Michael Sauder, "Rankings and Reactivity: How

Public Measures Recreate Social Worlds ", American Journal of Sociology, $\mathrm{n}^{\circ} 113,2007$, p. 1-40.

Fagot-Largeault, Anne, "Réflexions sur la notion de qualité de la vie ", Archives de philosophie du droit, tome 36, "Droit et Science ", 1991, p. 135-153.

Foucault, Michel, Naissance de la biopolitique, Cours du Collège de France (1978-1979), Paris, Gallimard/Seuil, 2004.

Foucault, Michel, Sécurité, territoire, population, Cours du Collège de France (1977-1978), Paris, Gallimard/Seuil, 2004.

Fourquet, François, Les Comptes de la puissance. Histoire de la comptabilité nationale et du Plan, Paris, Encres-Recherches, 1980.

Gadrey, Jean et Florence Jany-Catrice, Les Nouveaux indicateurs de richesse, Paris, La Découverte / Repères, 2005.

Hood, Christopher, "Contemporary Public Management: A New Paradigm? ", Public Policy and Administration, vol. 10, n 2, 1995, p. 104-117.

Hood, Christopher, "Control, Bargains, and Cheating: The Politics of Public-Service Reform ", Journal of Public Administration Research and Theory, vol. 12, n 3, 2002, p. 309-332.

Hopwood, Anthony et Peter Miller (dir.), Accounting as Social and Institutional Practice, Cambridge (UK), Cambridge University Press, 1994.

Laborier, Pascale et Dany Trom (dir.), Historicités de l'action publique, Paris, Presses universitaires de France, 2003.

Lascoumes, Pierre et Patrick Le Galès (dir.), Gouverner par les instruments, Paris, Presses de Sciences Po, 2004.

Latour, Bruno, Les Microbes. Guerre et paix, suivi de Irréductions, Paris, Métailié, 1984.

Lefrancq, Stéphane, "Recherche et action : la comptabilité dans son contexte. Une étude de la politique éditoriale d'Accounting, Organizations and Society ", Comptabilité-Contrôle-Audit, numéro thématique, juin 2004, p. 297-315.

Le Galès, Patrick, "Contrôle et surveillance. La restructuration de l'État en Grande-Bretagne », dans Pierre Lascoumes et Patrick Le Galès (dir.), Gouverner par les instruments, Paris, Presses de Sciences Po, 2004, p. 237-271.

Lie, Einar, "The Rise and Fall of Sampling Methods in Norway, 18751906 ", Science in Context, n 3, 2002, p. 385-409. 
Miller, Peter, "Accounting and Objectivity: The Invention of Calculating Selves and Calculable Spaces ", Annals of Scholarship, vol. 9, $\mathrm{n}^{\text {os }}$ 1-2, 1992, p. 61-86.

Miller, Peter et Timothy O'Leary, "Accounting and the Construction of the Governable Person ", Accounting, Organization and Society, vol. 12, $n^{\circ} 3,1987$, p. 235-265.

Morgenstern, Oskar, On the Accuracy of Economic Observations, Princeton, Princeton University Press, 1944, traduction française, L'Illusion statistique : précision et incertitude des données économiques, Paris, Dunod, 1950.

Nivière, Delphine, "Négocier une statistique européenne : le cas de la pauvreté ", Genèses, n 58, 2005, p. 28-47.

Pestre, Dominique, Introduction aux Science Studies, Paris, La Découverte / Repères, 2006.

Pezet, Eric (dir.), Management et conduite de soi. Enquête sur les ascèses de la performance, Paris, Vuibert, 2007.

Polanyi, Karl, The Great Transformation: The Political and Economic Origins of Our Time, Boston, Beacon Hill, 1944.

Porter, Theodore, Trust in Numbers. The Pursuit of Objectivity in Science and Public Life, Princeton, Princeton University Press, 1995.

Power, Michael, The Audit Society. Rituals of Verification, Oxford, Oxford University Press, 1999, traduction française, La Société de l'audit. L'obsession du contrôle, Paris, La Découverte, 2005.

Power, Michael, "Counting, Control and Calculation: Reflections on Measuring and Management ", Human Relations, vol. 57, n 6, 2004, p. $765-783$.

Prévost, Jean-Guy, A Total Science: Statistics in Liberal and Fascist Italy, Montréal, McGill Queen's University Press, 2009.

Salais, Robert, "La Politique des indicateurs. Du taux de chômage au taux d'emploi dans la stratégie européenne pour l'emploi (SEE) », dans Bénédicte Zimmermann (dir.), Action publique et sciences sociales, Paris, MSH, 2004, p. 287-331.

Supiot, Alain, L'Esprit de Philadelphie : la justice sociale face au marché total, Paris, Seuil, 2010.

Thévenot, Laurent, «Rules and Implements: Investments in Forms ", Social Science Information, vol. 23, $\mathrm{n}^{\circ} 1,1984$, p. 1-45, version française : "Les Investissements de formes ", Cahiers du Centre d'études de l'emploi, $\mathrm{n}^{\circ} 29$, Paris, Presses universitaires de France, 1986, p. 21-71.

Vanoli, André, Une Histoire de la comptabilité nationale, Paris, La Découverte, 2002, traduction anglaise, A History of National Accounting, 
Amsterdam, IOS Press, 2005.

Watts, Ross et Jerold Zimmerman, "Towards a Positive Theory of the

Determination of Accounting Standards ", The Accounting Review, vol. 53, $\mathrm{n}^{\circ} 1,1978$, p. 112-134. 